\title{
Marketization Plot of HTSC Maglev Mass Transportation in China
}

\author{
J. D. Fan ${ }^{\dagger}$, Hui Chen, Jin Yang and Guoqiang Chen
}

\begin{abstract}
The technology of high-temperature superconductive (HTSC) maglev trains has become mature and ready for a commercial use ten years after its first prototype was made by Jiasu Wang and Suyu Wang. However, an appropriate roadmap in applying it to the market is crucial for its success because superconductivity mystery remains veiled and public safety concern may prevent it from being used.
\end{abstract}

Keywords : HTSC Maglev Trains

\section{Introduction}

With the rapid development of economy the demand of high-speed transportation is boomed. Although air transportations provide us with high speed logistics, the limitation die to weather and transport capacity as well as safety concerns has greatly restricted their role in high speed transportations. One thus starts to turn to high-speed trains on the ground. However, the public concern of safety has been brought to the peak after the tailgated collision of CRH (China Railway High-Speed) motor train units on July 23, 2011, in China.

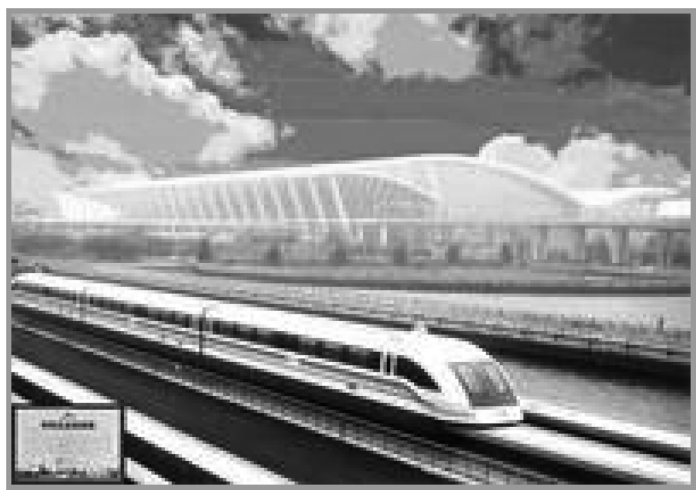

Fig. 1 A Conductor Maglev Train in Shanghai, China

\footnotetext{
$\dagger$ Corresponding author: Chongqing Academy of Science and Technology, Chongqing 401123, China

E-mail : Jdfan3@cox.net
}

We believe that this is the best period to promote the implementation of HTSC maglev trains. However, it is not so easy in implementing practice. China has already imported the technology of conductor maglev trains (CMT) from Germany and built up a $30 \mathrm{~km}$ long model maglev line to the Pudong international airport, Shanghai (Fig 1) [1]. CMT, abbreviated as "Maglev" in China, has deeply impressed Chinese society with extremely high costs. Whenever talking about HTSC maglev trains, audiences immediately recall Shanghai's "Maglev" and do not want to learn about details anymore. That is why we have to change the terminology to "HTSC Low-Air Trains."

\section{Technology of HTSC Maglev Trains}

\subsection{Principle of HTSC maglev trains}

The compound of $\mathrm{La}_{2-\mathrm{x}} \mathrm{Ba}_{\mathrm{x}} \mathrm{CuO}_{4-\mathrm{y}}$ discovered by J. G. Bednorz and k. A. Mueller [2] at Zurich Lab, IBM, in 1986, has opened a new era in the superconductivity community. Later, 1997, J. W. Chu's group, University of Houston, USA, had brought the critical temperature $\mathrm{T}_{\mathrm{c}}$ to about $95 \mathrm{~K}$ for a new superconductor of $\mathrm{YBa}_{2} \mathrm{Cu}_{3} \mathrm{O}_{7-\mathrm{x}}$, which makes applications of superconductors practical because cheapest liquid nitrogen easily reaches $77 \mathrm{~K}$. Moreover, one of the three characteristics of a superconductor - diamagnetism possibly leads to levitation of the superconductor under investigation in an applied magnetic field. It is just this property that is used in HTSC maglev transportation. 


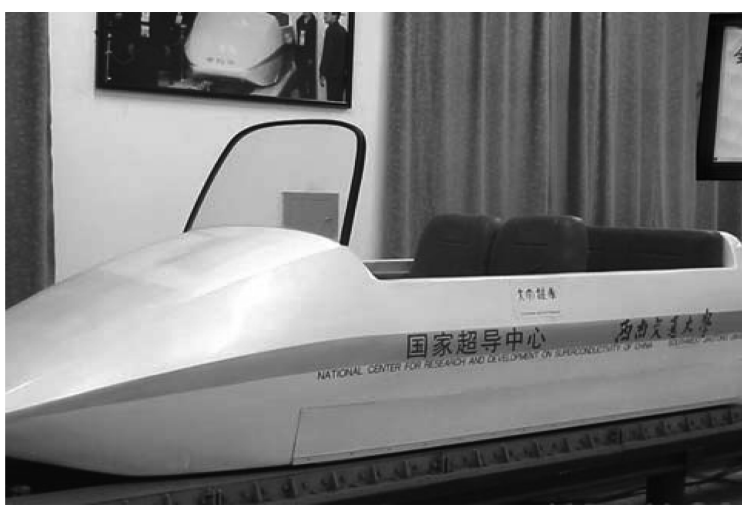

Fig. 2 Wangs' HTSC Maglev Train of “Century”

This characteristics used in HTSC maglev transportation is intrinsic of a superconductor, different to some extent from EM (electro-magnetic) levitation adopted in the Shanghai's maglev trains. Vehicles are levitated stably within the domain of the magnetic field without deviating from the balanced position. This technology greatly simplify the system of levitation, guidance and propelling of vehicles of the train, reduce the weight of each of vehicles and hence degrade the requirements for .road surface, leading to much lower costs in construction.

\subsection{China's HTSC maglev train projects}

As early as 2000, four years after the first HTSC sample was discovered, Professors Jiasu Wang and Suyu Wang have designed and manufactured their first HTSC maglev train prototype "Century" in the world (Fig. 2) [3].

The 0prototype has been put in their lab in a natural state of weather without appreciable changes in nature. During the past ten years, they have made great amount of progress in calculating, designing, and structuring an HTSC maglev train vehicle. Based on previously acquired technology on the measuring of important parameters of the HTS maglev system, they have extended this technology to develop new measurement systems with higher precision and more functional capabilities such as the study of dynamic characteristics of the HTS maglev system up to speeds of $300 \mathrm{~km} / \mathrm{h}$. Through these measurements many problems related to the HTS maglev; such as system optimization, stability control, influence from ramp angles, influence from AC field, and investigation of system's properties under $77 \mathrm{~K}$; have been studied,. Numerical calculations were also performed to confirm the validity of their experiments. Moreover, a 3D-model of the HTSC maglev was constructed via two different methods to simulate the maglev behavior, i. e. the behavior of an HTSC bulk sample(s) suspended over a permanent magnetic

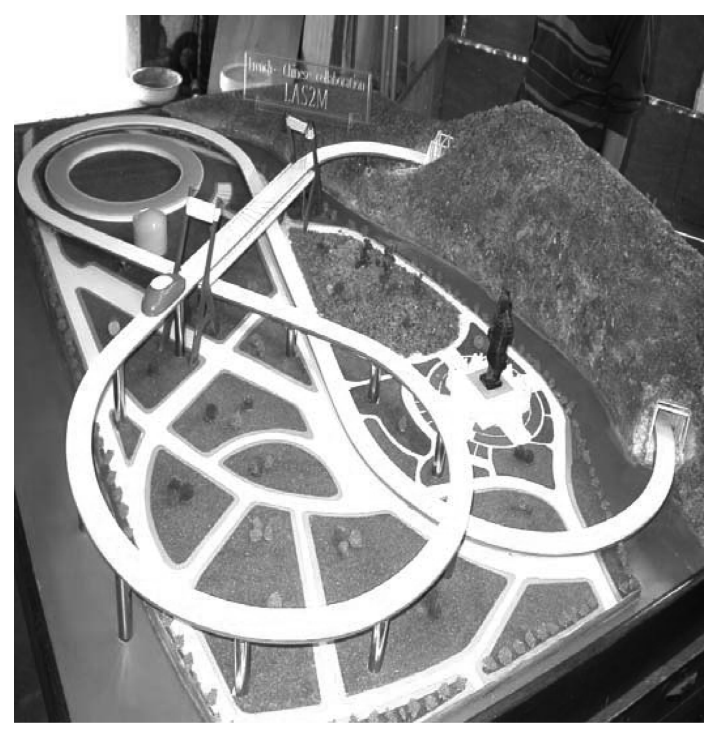

Fig. 3 Wanming Yang's Model HTSC Maglev Cars

guideway. Therefore, there are sufficient reasons to believe that their trial vehicle is able to be adopted in the case of low speed motion, such as an entertainment train system.

Besides Wangs' lab other professionals, such as Professor Yong Zhao, Southwest Jiaotong University, Chengdu, China; and Professor Wanming Yang, Shanxi Normal University, Xi'an, China, are also investigating the HTSC maglev train system in a small scale. Zhao's lab has a model maglev car on round permanent magnetic tracks with diameter of $5 \mathrm{~m}$. They plan to install a tunnel for it to mimic the low-vacuumed and tunneled high-speed transportation. Yang's work [4] is much earlier in model HTSC maglev cars (Fig. 3) that are very useful in education.

\section{Advantages of HTSC Maglev Trains}

The disaster of tailgated collision of two CRH motor train units occurred on July 23, 2011, in China alerted the public and officials of the importance and urgency of highspeed mass transportation safety. The HTSC maglev train systems we are promoting do possess advantages in two respects: (a) impossible to be in tailgated collisions, (b) impossible to be out of rails.

The reason why two vehicles may collide is the two propelling systems independent from each other. Now, for the HTSC maglev train systems there is no propelling \& braking system at all inside any vehicle. They are propelled and broken by linear motors installed on the ground. That means that they are in motion synchronically. One can divide the rail line into sections to supply power for the linear motors. Once a vehicle stops for whatever cause, the 
other entering the section supplied with power will lose power as well and moves because of inertia and eventually stops. As long as the rail section is long enough for a train without propelling to stop, two vehicles can never collide. In addition, if one adds safe wheels to each of the vehicles like an airplane when an emergent stop is needed, the stopping distance will be much short, so will be the length of each section of the rail line. It can therefore save power and operation costs.

Moreover, since a train is supported by a magnetic force produced by the permanent magnets of rack rails and moving along the magnetic field. Even if a part of the rails is damaged or removed, the train remains to move forward along the field without being out of racks, in fact, the magnetic field only if the damaged part is far shorter than the length of the vehicle. This is because the damaged part of rails leads to a reduction of the levitation force and hence the rising height due to a less supporting force that exponentially increases with the decrease of the levitation height, which compensates the loss of a part of the supporting force to the vehicle. When a train is moving at a high speed, it passes this short damaged section in a several tens milliseconds. Passengers may even not feel any difference but a little jerk.

In addition, because there is neither propelling and braking system nor device producing an EM field like Shanghai's CMT, the vehicle is much lighter and can be made of aluminum or magnesium to further reduce its own weight. As a result, the requirements for road construction would be much degraded. This will in trun greatly reduce the whole project costs very much.

\section{Marketization Plot}

\subsection{Current situation in china}

It is for sure that with the technology developed in China it is too early to construct a practically usable HTSC maglev mass transportation line. Clearly, tests and examinations of safety and stability of a train system at as high speed as $200 \mathrm{~km} / \mathrm{h}$ or above are needed. In turn, these tests and examinations need a great amount of fund. To be sure for safety, etc. it is a must to make a large amount of investments in research. However, most of investors can only put money into the projects reliable and fully trustful. The work to persuade government officials and investors is however too hard to any of scientists and engineers. In addition, competition in research funding also prevents people from being fair has greatly delay its process as well.

However, we believe that the current technology is good enough to implement a project for education and entertain- ments. At low speed, such as $20 \mathrm{~km} / \mathrm{h}$, safety issue is not the major concern. Thus, we can get the technology go out of laboratory, letting regular people be aware of this new technology. Now, Yang's model maglev trains can seemingly be made for educational purpose of HTSC maglev technology in high schools and colleges. Wangs' "Century" can be extended to design a new type of entertainment. The public awareness will greatly enhance their support and governmental decision of making large investments in research of this advanced high-tech mass transportation systems.

\subsection{A roadmap of implementing marketization}

We have worked hard to push forward this project. First of all, since the technology remains in laboratory to date we cannot dream of immediate welcome from mass transportation sectors and public. It is is necessary to do pilot tests at higher speed before the technology can be used for constructing a section of HTSC railroad. However, it is possible for it to be used in entertainments and public education \& science popularization. A roadmap for this huge project is suggested as follows.

a) Producing model maglev cars for public awareness and fund-raising.

b) Constructing an entertainment line of HTSC maglev trains in a touring zone for public awareness and fund-raising.

c) Renovating an old or abandoned railroad for high speed pilot tests to save funds.

d) Constructing a special line to the local airport for mass transportation.

e) Building an inter-city line for mass transportation.

Chongqing or any of the large cities in China has the capacity and ability of setting up such a project for adding one more point of economic growth and developing one of high-techs in the national economy.

\section{References}

1. Shanghai Maglev Transportation Development Co. Ltd. www.smtdc.com/ and http://economy.enorth.com.cn/ system/ 2006/04/27/001291035.shtml.

2. J. G. Bednorz and K. A. Mueller (1986). Z. Phys. B-Cond. Matt. Vol. 64, p. 189.

3. Wang Jia-Su, et al. (2009). Journal of Low temperature Physics, Vol. 31, No. 5, pp. 42-15.

4. W. M. Yang, et al. (2006). Supercond. Sci. Technol. Vol. 19, pp. S537-S539.

Received(July 5, 2012), Accepted(July 30, 2012) 